\title{
Carfilzomib for relapsed and refractory multiple myeloma
}

This article was published in the following Dove Medical Press journal: Cancer Management and Research

K Groen

NWCJ van de Donk CAM Stege

S Zweegman

IS Nijhof

Department of Hematology, VU University Medical Center, Amsterdam, Netherlands
Correspondence: K Groen

Department of Hematology, VU

University Medical Center, III7

De Boelelaan, Amsterdam I08IHV,

Netherlands

Tel +3I 204442604

Fax +3I 204444260 I

Email k.groen I@vumc.nl

\begin{abstract}
Although the prognosis of multiple myeloma (MM) patients has dramatically improved during recent years, virtually all patients eventually develop relapsed refractory disease. Several new therapeutics have been developed in the last few years, including carfilzomib, a second-generation proteasome inhibitor (PI) that has been approved by the US Food and Drug Administration (FDA) in the setting of relapsed and/or refractory MM, as a single agent with or without dexamethasone, and in combination with lenalidomide in 2012 and 2015, respectively. Other promising combinations with carfilzomib are being investigated. Carfilzomib has shown superiority over the first-generation PI bortezomib on both efficacy and toxicity. In particular, profoundly lower incidence in polyneuropathy compared to bortezomib has been described. However, carfilzomib has a different toxicity profile, with more cardiovascular adverse events. Therefore, caution should be taken with the use of carfilzomib for elderly and cardiovascularly compromised patients. The once-weekly administration of carfilzomib, recently approved by the FDA in combination with dexamethasone, will lead to a lower burden for the patient and caregivers compared to the twice-weekly schemes that were routinely used until recently. This review has a focus on clinical trial data that has led to drug approval, as well as new promising combination studies, and provides advice for treating physicians who are now prescribing this drug to patients.
\end{abstract}

Keywords: carfilzomib, relapsed, refractory multiple myeloma, proteasome inhibitor

\section{Introduction}

Outcomes for multiple myeloma (MM) patients have improved significantly during recent years, mainly due to the application of high-dose conventional therapy with autologous stem-cell transplantation as a routine procedure for transplant-eligible MM patients, significant improvements in supportive care strategies, and the introduction and widespread use of the immunomodulatory imide drugs (IMiDs) thalidomide and lenalidomide and the proteasome inhibitor (PI) bortezomib. ${ }^{1}$ However, the majority of patients eventually develop relapsed and refractory disease, and the prognosis of MM patients who have received at least three prior lines of therapy who have become double-refractory to IMiDs (thalidomide or lenalidomide) and PIs (bortezomib) and have been exposed to an alkylating agent is very poor, with eventfree survival and overall survival (OS) only 5 and 13 months, respectively. ${ }^{2}$ Therefore, the development of new therapeutics, especially for this group of patients, is needed.

Proteasome inhibition is a highly effective treatment for MM. Almost 15 years ago, the US Food and Drug Administration (FDA) approved the first PI, bortezo- 
mib, for the treatment of relapsed and/or refractory MM (RRMM) and subsequently for frontline MM treatment. The introduction of bortezomib led to improved progression-free survival (PFS) and OS of MM patients. ${ }^{3}$ Nevertheless, the presence of primary resistance or development of acquired resistance to bortezomib is common, and treatment is often limited by dose-limiting side effects, most often due to peripheral neuropathy (PNP). Although subcutaneous administration instead of intravenous administration decreases the incidence of PNP, $\sim 5 \%-10 \%$ of patients still experience severe PNP. Therefore, second-generation PIs were developed to improve efficacy, combined with a different toxicity profile. Carfilzomib is a new-generation PI, which in contrast to the reversible binding of bortezomib, binds irreversibly and selectively to its target: the chymotrypsin-like activity of the 20 S proteasome. In 2012 , the FDA approved carfilzomib for the treatment of patients who have received at least two prior therapies, including bortezomib and an immunomodulatory agent, and who have demonstrated disease progression on or within 60 days of the completion of last therapy. ${ }^{4-6}$

\section{Mechanisms of action}

The proteasome is a multienzyme catalytic complex found in the nucleus and cytoplasm of eukaryotic cells that is responsible for degrading or processing intracellular proteins.
Ubiquitylation of proteins marks the proteins for proteasomal degradation. Proteasome inhibition leads to accumulation of intracellular proteins, resulting in cell death. ${ }^{7,8}$

The $20 \mathrm{~S}$ proteasome consists of 28 protein subunits, in a cylindrical structure, created by four stacked rings. Two of those rings consist of seven $\alpha$-subunits and two rings consist of seven $\beta$-subunits. Proteasome enzymatic activities are performed by three of the seven $\beta$-subunits: $\beta_{1}, \beta_{2}$, and $\beta_{5}$. These proteasome enzymatic activities have been characterized as chymotrypsin-like. Bortezomib is a slowly reversible inhibitor, targeting the chymotrypsin-like activity of the proteasome. Compared to bortezomib, carfilzomib has greater selectivity for the chymotrypsin-like activity of the proteasome and binds irreversibly. Carfilzomib administration leads to dosedependent inhibition of the chymotrypsin-like proteasome activity in all tissue, except for the brain. Figure 1 shows the mechanism of proteasome inhibition by carfilzomib. ${ }^{4,9}$

Carfilzomib selectively inhibits the $\beta_{5}$ subunit of the constitutive proteasome (c20S) and LMP7 of the immunoproteasome (i20S). Inhibition of all proteasome subunits leads to cytotoxic effects in hematologic tumor cells, but also to peripheral blood mononuclear cells. When selectively inhibiting $\beta_{5}$ and LMP7, an antitumor effect is seen, though with minimal toxicity in untransformed cells. With MM cells, inhibition of chymotrypsin-like subunits alone is enough to induce apoptosis by inducing proteasome-substrate

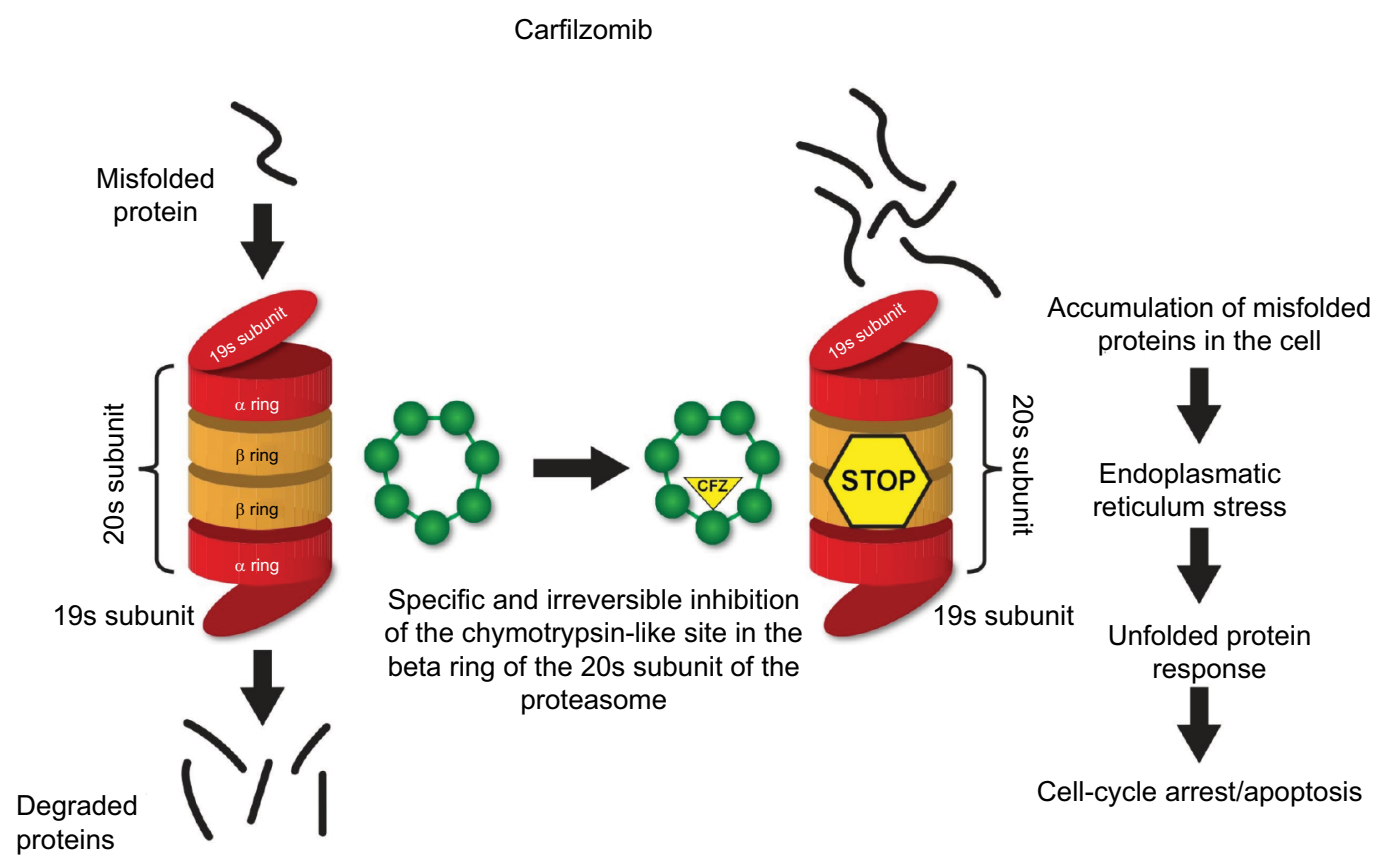

Figure I Mechanism of proteasome inhibition by carfilzomib.

Notes: This figure has been adapted from an article originally published in ASH News and Reports. Kortuem KM and Stewart AK. Carfilzomib. Blood. 2013; I2I:893-897. @ the American Society of Hematology. 
accumulation. This might be the reason that MM cells are so sensitive to chymotrypsin-like subunits. ${ }^{10}$

In contrast to bortezomib, carfilzomib is associated to a lesser extent with treatment-induced PNP. Bortezomibinduced PNP might be explained by a non-proteasomedependent mechanism, and this drug inhibits also several nonproteasome targets (eg, serine proteases cathepsin G, cathepsin A, rennin, dipeptidyl peptidase II, and $\mathrm{HtrA} 2 / \mathrm{Omi}$ ). HtrA2/Omi is known to be involved in neuronal survival, and is inhibited by bortezomib, but not by carfilzomib. This may explain why the unselective binding of bortezomib seems to play an important role in the high rates of sensory polyneuropathy associated with this drug. ${ }^{11}$

Carfilzomib has good penetration throughout the body, but does not cross the blood-brain barrier. It has a very short half-life of $\sim 30$ minutes, and is metabolized extrahepatically into inactive metabolites. This means carfilzomib is not dependent on liver function, and interactions with hepatically cleared comedication are uncommon. This in contrast to bortezomib, which is mostly metabolized in the liver. ${ }^{12}$

This review provides an overview of the most important phase I, II, and III studies concerning carfilzomib in different combinations, describing efficacy and toxicity. Finally, we discuss possible treatment options for patients with RRMM, with the focus on carfilzomib-based regimens. We also provide guidelines for the clinical management of carfilzomib.

\section{Carfilzomib monotherapy}

Several dosing strategies were investigated in phase I trials, which are summarized in Table 1. The maximum tolerated

Table I Clinical trials performed with carfilzomib, including ORR and survival data

\begin{tabular}{|c|c|c|c|c|c|c|c|}
\hline Trails & $\begin{array}{l}\text { First } \\
\text { author }\end{array}$ & Phase & $\begin{array}{l}\text { Patients, } \\
\text { n }\end{array}$ & ORR & CR + VGPR & $\begin{array}{l}\text { Median PFS } \\
\text { (months) }\end{array}$ & $\begin{array}{l}\text { Median OS } \\
\text { (months) }\end{array}$ \\
\hline$P X-|7|-\left.00\right|^{14}$ & O'Connor & 1 & 10 & $20 \%$ & NR & NR & NR \\
\hline PX-171-002 & Alsina & $\mathrm{I}$ & $21+7$ & NR & NR & NR & NR \\
\hline PX-|17I-003-A| ${ }^{16}$ & Siegel & II & 266 & $23.7 \%$ & $5.5(0.4+5.1)$ & 3.7 & 15.6 \\
\hline PX-17I-004 & $V_{i j}$ & II & $129 *$ & $\begin{array}{l}42.4 \% \text { and } \\
52.2 \%\end{array}$ & $\begin{array}{l}17(3.4+13.6) \text { and } \\
28.4(1.5+26.9)\end{array}$ & 8.2 vs NR & NR \\
\hline Carfilzomib in bortezomib exposed ${ }^{18}$ & $V_{i j}$ & II & 35 & $17.1 \%$ & $5.7(2.8+2.8)$ & 4.6 & 29.9 \\
\hline Carfilzomib in 30 minutes infusion ${ }^{21}$ & Papadopoulos & $\mathrm{I}$ & 33 & $48 \%-60 \%$ & $21(4+17)$ & $6.2-7$ & NR \\
\hline $\begin{array}{l}\text { Carfilzomib } 56 \mathrm{mg} / \mathrm{m}^{2} \mathrm{w} / \mathrm{wo} \\
\text { dexamethasone }\end{array}$ & Lendvai & II & 42 & $55 \%$ & $23(2+2 I)$ & 4.1 & 20.3 \\
\hline ENDEAVOR $^{24}$ & Dimopoulos & III & 929 & $\begin{array}{l}77 \% \text { vs } \\
63 \%\end{array}$ & $\begin{array}{l}54(13+42) \text { vs } 29 \\
(6+22)\end{array}$ & 18.7 vs 9.4 & 47.6 vs 40.0 \\
\hline PX-I7I-006, phase $I b^{25}$ & Niesvizky & IB & 40 & $62.5 \%$ & $35(2.5+32.5)$ & 10.2 & NR \\
\hline PX-I7I-006, phase $2^{26}$ & Wang & II & 52 & $76.9 \%$ & $42.2(5.7+36.5)$ & 15.4 & NR \\
\hline ASPIRE $^{27}$ & Stewart & III & 792 & $\begin{array}{l}87.1 \% \text { vs } \\
66.7 \%\end{array}$ & $\begin{array}{l}69.9(31.8+38.1) \text { vs } \\
40.4(9.3+31.1)\end{array}$ & 26.1 vs 16.6 & 48.3 vs 40.4 \\
\hline CHAMPION-I ${ }^{38}$ & Berenson & $1 / 11$ & 104 & $77 \%$ & $47(14+33)$ & 12.6 & NR \\
\hline $\mathrm{ARROW}^{13}$ & Moreau & III & 478 & $\begin{array}{l}62.9 \% \text { vs } \\
40.8 \%\end{array}$ & $\begin{array}{l}34(7+27) \text { vs } 13 \\
(2+11.7)\end{array}$ & II. 2 vs 7.6 & $\begin{array}{l}\text { Not yet } \\
\text { mature }\end{array}$ \\
\hline Ibrutinib and carfilzomib ${ }^{34}$ & Chari & $\mathrm{I}$ & 42 & $67 \%$ & $23(2+2 I)$ & 7.2 & Not reached \\
\hline Ibrutinib and carfilzomib ${ }^{35}$ & Chari & II & 36 & $72 \%$ & $28(6+22)$ & 11.6 & NR \\
\hline PX-|7|-0II: FOCUS ${ }^{20}$ & Hájek & III & 315 & $\begin{array}{l}19.1 \% \text { vs } \\
11.4 \%\end{array}$ & $4(I+3)$ vs $3(0+3)$ & 3.7 vs 3.3 & 10.2 vs 10.0 \\
\hline $\begin{array}{l}\text { Carfilzomib, pomalidomide, and } \\
\text { dexamethasone }{ }^{33}\end{array}$ & Shah & $\mathrm{I}$ & 32 & $50 \%$ & $16(0+16)$ & 7.2 & 20.6 \\
\hline $\begin{array}{l}\text { Carfilzomib, lenalidomide, vorinostat, and } \\
\text { dexamethasone }{ }^{32}\end{array}$ & Vesole & $\mathrm{I}$ & 17 & $53 \%$ & $12(0+12)$ & 12 & Not reached \\
\hline Panobinostat and carfilzomib ${ }^{30}$ & Berdeja & $\mathrm{I} / \mathrm{II}$ & 42 & $67 \%$ & 33 VGPR or better & 7.7 & Not reached \\
\hline Panobinostat and carfilzomib ${ }^{31}$ & Kaufman & $\mathrm{I}$ & 20 & $50 \%$ & 20 VGPR or better & 14.3 & NR \\
\hline Isatuximab and carfilzomib ${ }^{36}$ & Chari & IB & 29 & $66 \%$ & $27.5(3.4+24.1)$ & Not reached & NR \\
\hline Isatuximab and carfilzomib ${ }^{47}$ & Martin & IB & 11 & $80 \%$ & $18.2(0+18.2)$ & NR & NR \\
\hline $\begin{array}{l}\text { MMYI00I: daratumumab with carfilzomib } \\
\text { and dexamethasone in lenalidomide- } \\
\text { refractory patients }{ }^{37}\end{array}$ & Chari & IB & 51 & $81 \%$ & $68(12+56)$ & 14.1 & NR \\
\hline
\end{tabular}

Note: *Cohort I, $20 \mathrm{mg} / \mathrm{m}^{2}$; cohort 2, intravenous $20 / 27 \mathrm{mg} / \mathrm{m}^{2}$.

Abbreviation: CR, complete response; NR, not reported; ORR, overall response rate; OS, overall survival; PFS, progression-free survival; VGPR, very good partial response. 
dose (MTD) was not reached, and the maximum dose tested was $20 \mathrm{mg} / \mathrm{m}^{2}$ for the first two doses, which was followed by a higher dose of $27 \mathrm{mg} / \mathrm{m}^{2}\left(20 / 27 \mathrm{mg} / \mathrm{m}^{2}\right)$. This dosing regimen was used in subsequent clinical trials. However, there is still uncertainty about the most optimal dosing scheme, as exemplified by the recent ARROW study comparing once-weekly administration of carfilzomib at a higher dose vs twice-weekly lower dosing, which is discussed later. ${ }^{13-15}$

Carfilzomib was tested in a phase II study (PX171-003-A1) by Siegel et al in patients with RRMM. Carfilzomib was dosed at $20 \mathrm{mg} / \mathrm{m}^{2}$ in the first cycle to abrogate potential tumor-lysis syndrome (TLS). Thereafter, dosing was escalated to $27 \mathrm{mg} / \mathrm{m}^{2}$ in cycle 2 and further cycles. In this study, dexamethasone $4 \mathrm{mg}$ was given as premedication on the days of carfilzomib. A total of 266 heavily pretreated MM patients participated in this study, with median prior lines of therapy $5 \%$ and $80 \%$ of patients double-refractory. A maximum of 12 cycles were given, with $15 \%$ of patients reaching this point; $59 \%$ of patients discontinued treatment early due to progressive disease, $12 \%$ due to adverse events (AEs).

The overall response rate (ORR) was $23.7 \%$, with a clinical benefit rate (CBR) of $37.0 \%$. Patients who were refractory to bortezomib in their last line of therapy had an ORR of $18.6 \%$, compared to $28.3 \%$ for patients who did not receive bortezomib in their most recent line of therapy. The duration of response (DOR) was 7.8 months for patients who had a partial response (PR) or better. Median PFS was 3.7 months, with median OS of 15.6 months, for all response-evaluable patients. Outcome was not influenced by adverse cytogenetics, renal impairment, or Eastern Cooperative Oncology Group performance score. Although $77 \%$ of patients already had grade 1 or 2 PNP at baseline, only $12.4 \%$ experienced new or progression of preexisting PNP, with only $1.1 \%$ of patients experiencing grade 3 PNP. Overall, this phase II study with carfilzomib monotherapy was very tolerable and showed notable response in a heavily pretreated group of MM patients. ${ }^{16}$

Another phase II study (PX-171-004) was conducted by Vij et al in which RRMM but bortezomib-naïve MM patients were treated with carfilzomib monotherapy. The study started with 59 patients who received carfilzomib at a dose of $20 \mathrm{mg} /$ $\mathrm{m}^{2}$ without dose escalation. However, due to encouraging data from the PX-171-002 study, this study was amended, and a second cohort of 70 patients received a dose of $27 \mathrm{mg} /$ $\mathrm{m}^{2}$ from the second cycle onward.

Enrolled patients had received a median of two prior lines of therapy, including steroids, immunomodulatory agents, and autologous stem-cell transplants. Cohort 1 showed an ORR of
$42.4 \%$, which increased to $52.2 \%$ in cohort 2 . The CBR was $59.3 \%$ and $64.2 \%$ respectively. Median DOR was 13.1 months in cohort 1, with median PFS of 8.2 month. Neither of these was reached in cohort 2, with a median follow up of 11.5 months. One patient in cohort 1 experienced grade 3 PNP, and none in cohort 2 . No patients discontinued treatment because of PNP. Overall, treatment-related AEs were similar to previous studies. This study showed promising results for carfilzomib as a single agent in a pretreated but bortezomib-naïve MM-patient population, especially taking into consideration that more than a third of the patients were able to continue treatment for $>12$ months without significant toxicity. ${ }^{17}$

Vij et al also conducted a carfilzomib phase II study in which RRMM patients had been previously treated with bortezomib. In this study, median prior lines of treatment was seven. All 35 patients received carfilzomib twice weekly at $20 \mathrm{mg} / \mathrm{m}^{2}$. The ORR was substantially lower, $17.1 \%$, with a CBR of $31.4 \%$. AE rates were comparable to the PX-171-003 study. ${ }^{18}$ The impact of renal insufficiency in carfilzomib treatment was investigated in a phase II trial with 50 relapsed MM patients with varying renal impairment. Also here, toxicity was manageable and independent of renal status. Dose adjustments were not necessary. ${ }^{19}$

The PX-171-003-A1 study led to FDA approval of carfilzomib monotherapy for patients with RRMM with progression during or after therapy with bortezomib and an immunomodulatory derivate (IMiD). However the European Medicines Agency (EMA) required a phase III study in order to approve carfilzomib as a single agent. Therefore, the randomized phase III FOCUS study was initiated, which compared carfilzomib monotherapy $\left(20 / 27 \mathrm{mg} / \mathrm{m}^{2}\right)$ with low-dose corticosteroids combined with optional cyclophosphamide in patients with at least three prior lines of therapy. ${ }^{16}$

In the FOCUS study (phase III, PX-171-011) by Hájek et al in 2017, patients with RRMM were treated with carfilzomib $20 / 27 \mathrm{mg} / \mathrm{m}^{2}$ ) or low-dose corticosteroids, with optional cyclophosphamide in the control arm. Low dose corticosteroids consisted of dexamethasone $6 \mathrm{mg}$ or prednisone $30 \mathrm{mg}$ every other day or another equivalent corticosteroid dose. Additional cyclophosphamide (50 mg) was optional, but was given to $95 \%$ of patients in the control arm.

The primary end point was OS, with PFS, ORR, DOR, $\mathrm{CBR}$, and disease-control rate as secondary end points. A total of 157 patients participated in the carfilzomib group vs 158 in the control group. The ORR was $19.1 \%$ and $11.4 \%$, respectively, which was significant $(P=0.0305)$, but this was not translated into superior median PFS (3.7 months in the carfilzomib group vs 3.3 months in the control group, 
$P=0.2479)$ or median DOR (7.2 months and 9.5 months, respectively). Median OS was comparable, with 10.2 months in the carfilzomib group vs 10.0 months in the control group; therefore, the study did not meet the expected primary objective of carfilzomib superiority in OS over the control arm. Not meeting the primary end point of this study was most probably due to not adding dexamethasone to carfilzomib and the high efficacy of additional cyclophosphamide in the control group, which enhanced expected ORR and PFS in the control arm.

Grade 3 or higher AEs were comparable in both groups, except for acute renal failure (carfilzomib 8\%, control 3\%) and pneumonia (carfilzomib 6\%, control 12\%). Furthermore, cardiac failure of any grade was seen in seven patients in the carfilzomib group vs one patient in the control group. There was no difference in reported PNP between the groups. ${ }^{20}$

\section{Approved carfilzomib-combination therapy}

It is generally known that combination therapy has a stronger antimyeloma effect compared to monotherapy. However, combination therapies might lead to higher toxicity rates. In this section, we describe the studies that led to the approval of two combination therapies with carfilzomib (carfilzomib plus dexamethasone and carfilzomib combined with lenalidomide and dexamethasone).

\section{Carfilzomib and dexamethasone}

In 2014, Papadopoulos et al conducted a phase I study in which the infusion time of carfilzomib was extended from 2 to 10 to 30 minutes, allowing higher doses of carfilzomib. Dosing started at $20 \mathrm{mg} / \mathrm{m}^{2}$ again, though with dose escalation to $36,45,56$, and $70 \mathrm{mg} / \mathrm{m}^{2}$. The study was amended to investigate the safety and efficacy of carfilzomib combined with low-dose dexamethasone (40 mg weekly). The MTD was established at $56 \mathrm{mg} / \mathrm{m}^{2}$. The ORR for carfilzomib monotherapy was $48 \%$, with a CBR of $52 \%$. Adding dexamethasone resulted in an ORR of $55 \%$ and CBR of $64 \%$. The safety profile in this study was comparable to previous phase I/II trials with carfilzomib. ${ }^{21}$

In 2014, a single-arm/single-center phase II study was published by Lendvai et al in which patients received carfilzomib $\left(20 / 56 \mathrm{mg} / \mathrm{m}^{2}\right)$. After two cycles of carfilzomib monotherapy, dexamethasone $20 \mathrm{mg}$ could be added if response was less than PR. Dexamethasone could also be added at the time of progression. A total of 44 patients participated, with 42 evaluable for response: 17 patients achieved a PR or better with single-agent carfilzomib, while 6 of these 17 patients progressed later in treatment and received additional dexamethasone. A total of 23 of the 42 patients achieved a PR or better (55\% ORR), with a median time to best response of two cycles and median DOR of 11.7 months. Median PFS and OS were 4.1 months and 20.3 months, respectively. There was no difference in outcome between cytogenetic high-risk and non-high-risk patients. Grade 3 or 4 AEs were lymphopenia $(43 \%)$, thrombocytopenia (32\%), leukopenia (18\%), anemia (18\%), and neutropenia (18\%). Grade three or four non-hematologic AEs were hypertension (25\%), pneumonia $(18 \%)$ and heart failure (11\%). PNP occurred in six patients, all grade 1, with two experiencing a worsening of preexisting PNP. Adding dexamethasone did not seem to have an effect on type or rate of AEs. ${ }^{22}$

Based on these promising results, the phase III ENDEAVOR study was initiated, which evaluated carfilzomib and dexamethasone vs bortezomib and dexamethasone in 929 RRMM patients with one to three prior lines of therapy. Importantly, bortezomib-refractory patients were excluded from trial participation. Carfilzomib was started at a dose of $20 \mathrm{mg} / \mathrm{m}^{2}$ for the first 2 days in cycle 1 , and $56 \mathrm{mg} / \mathrm{m}^{2}$ thereafter. Bortezomib was given twice weekly subcutaneously or intravenously.

Dimopoulos et al wrote an interim analysis of the primary end point of this study. There was a significant benefit in terms of PFS for the carfilzomib-treated patients when compared to the control group (median PFS 18.7 vs 9.4 months, $P<0.0001$ ). The objective ORR was $77 \%$ in the carfilzomib group vs $63 \%$ in the bortezomib group $(P<0.0001)$, with DOR 21.3 months vs 10.4 months, respectively. The median time to response was 1.1 months in both groups. Although there was an improvement in PFS in both high-risk and standard-risk patients, carfilzomib could not totally overcome the negative impact of high-risk cytogenetics on PFS in this study.

In this analysis, OS data were not yet mature. However, in 2017 an updated analysis with prolonged follow-up showed a marked improvement in median OS of 47.6 months in the carfilzomib group vs 40.0 months in the bortezomib group $(P=0.010) .{ }^{23}$ The most common AEs (grade 3 or higher) were anemia (14\% vs 10\%), hypertension ( $9 \%$ vs $3 \%$ ), thrombocytopenia ( $8 \%$ vs $9 \%$ ), and pneumonia ( $7 \%$ vs $8 \%$ ). PNP (grade 2 or higher) was seen in $6 \%$ of patients in the carfilzomib group compared to $32 \%$ in the bortezomib group. In both groups, $5 \%$ of patients died during treatment within 30 days of last administration. In 151 patients, divided over both groups, serial echocardiography performed, which showed two patients in both groups with reduction in their left ventricular ejection fraction. ${ }^{24}$ 
In conclusion, the ENDEAVOR study showed a benefit for carfilzomib-dexamethasone compared to bortezomibdexamethasone in patients with one to three prior lines of therapy, with an important reduction in PNP. In this study, there was no benefit of serial echocardiography in preventing cardiovascular events. ${ }^{24}$ These data led to both FDA and EMA approval of this regimen.

\section{Carfilzomib, lenalidomide and dexamethasone}

In the PX-171-006 study, carfilzomib was combined with lenalidomide and low-dose dexamethasone, assessing safety and tolerability in 40 patients. Carfilzomib was given twice weekly starting at a dose of $15 \mathrm{mg} / \mathrm{m}^{2}$, which was gradually enhanced to $27 \mathrm{mg} / \mathrm{m}^{2}$. Lenalidomide was given in the first 21 days of a 28-day cycle, ranging from $10 \mathrm{mg}$ a day to 25 $\mathrm{mg}$ a day. Dexamethasone was given at a dose of $40 \mathrm{mg}$ weekly. The MTD was not reached, and thus the maximum planned dose (MPD) of the phase I study was used in the phase II study. Grade 3 or 4 hematologic toxicities were neutropenia $(42.5 \%)$, anemia $(20 \%)$, thrombocytopenia (32.5\%), lymphopenia (27.5\%), and nonhematologic toxicities of grade 3 or 4 were fatigue $(7.5 \%)$, diarrhea $(5.0 \%)$, hyperglycemia $(22.5 \%)$, hypokalemia (10.0\%), hyponatremia (15\%), and hypophosphatemia (20\%). Neuropathic events were reported in only $10 \%$ of patients, all grade 1 or 2, and all these patients had a history of neuropathy. The ORR was $62.5 \%$, with a CBR of $75 \%$. Median DOR was 11.8 months for patients who achieved at least a PR. Median PFS was 10.2 months. ${ }^{25}$

This led to the phase II dose-expansion study (PX171-006) investigating safety and secondary end points as ORR and DOR, with 52 patients treated at the MPD. The ORR was $76.9 \%$, with a median DOR of 22.1 months. Median time to response was 0.95 month, and median PFS 15.4 months. Median DOR was also 22.1 months for patients refractory to bortezomib (13 patients), with ORR of $69.2 \%$ and median PFS of 15.4 months. In lenalidomide-refractory patients, the ORR was $69.9 \%$, with median DOR of 10.8 months and median PFS of 7.9 months. In the MPD cohort, $94.2 \%$ of patients experienced a grade 3 or $4 \mathrm{AE}$, including neutropenia (32.7\%), thrombocytopenia (19.2\%), anemia (19.2\%), and lymphopenia (48.1\%). The most common AEs of any grade were fatigue $(69.2 \%)$ and diarrhea $(57.7 \%)$, both mostly grade 1 or 2 . Only one patient experienced grade 3 neuropathy. A total of $19.2 \%$ of patients had a cardiac AE of any grade, with three patients experiencing a grade 3 or higher AE. ${ }^{26}$
Based on these studies, Stewart et al performed a phase III study (ASPIRE) in which the effect of adding carfilzomib to lenalidomide and dexamethasone was investigated in RRMM patients with one to three prior lines of therapy. In this study, carfilzomib dosed at $20 \mathrm{mg} / \mathrm{m}^{2}$ on days 1 and 2 of the first cycle and $27 \mathrm{mg} / \mathrm{m}^{2}$ thereafter was used (28day cycles, carfilzomib given on days $1,2,8,9,15$, and 16) with 2-10 minutes' infusion. A total of 792 patients were included in the study, equally divided over the two groups. Median PFS was 26.1 months in the carfilzomib group compared to 16.6 months in the control group $(P<0.001)$. Median OS was 48.3 months vs 40.4 months, respectively $(P=0.0045)$. The difference in OS was more pronounced in patients treated with one prior line of therapy (11.4 months) compared to those treated with two or more prior lines of therapy (6.5 months).

The 24-month OS was $73.3 \%$ in the carfilzomib group compared to $65.0 \%$ in the control group, and ORR $87.1 \%$ and $66.7 \%(P<0.0001)$ with a complete response rate of $31.8 \%$ and $9.3 \%(P<0.0001)$, respectively. Median time to response was 1.6 months for the carfilzomib group and 2.3 months in the control group, with median DOR of 28.6 months vs 21.2 months, respectively. Also, quality of life improved significantly more in the carfilzomib group. Adding carfilzomib to lenalidomide-dexamethasone did not cause an increase in PNP incidence (17.1\% vs $17.0 \%$ ). In the carfilzomib group, any grade $3 \mathrm{AE}$ was reported in $83.7 \%$ of patients compared to $80.7 \%$ in the control group: cardiac failure happened in $3.8 \%$ vs $1.8 \%$, ischemic heart disease in $3.3 \%$ vs $2.1 \%$, hypertension in $4.3 \%$ vs $1.8 \%$, dyspnea in $2.8 \%$ vs $1.8 \%$, and acute renal failure in $3.3 \%$ vs $3.1 \%$, respectively. In both groups, $6.9 \%$ of patients died as a result of an AE. Adding carfilzomib to lenalidomide and dexamethasone has led to a significantly improved PFS of 7.9 months, with improved quality of life and an acceptable toxicity profile. ${ }^{27,28}$

\section{Other new, non-FDA-approved combinations with carfilzomib} Carfilzomib and deacetylase inhibitors

Deacetylase inhibitors like panobinostat and vorinostat have shown synergy with PIs like bortezomib. The mechanism of synergy is likely multifactorial, including disruption of protein degradation and inhibition of the interaction of MM cells with their microenvironment. ${ }^{29}$ In 2015, a phase I/II trial was published investigating the MTD of carfilzomib in combination with the deacetylase inhibitor panobinostat in patients with RRMM. A total of 44 patients participated: 13 in phase I and 31 in phase II. Panobinostat was administered 
on days $1,3,5,15,17$, and 19 of a 28-day cycle, carfilzomib on days $1,2,8,9,15$, and 16 . No dose-limiting toxicity (DLT) was observed, and thus the MTD in this study was defined as panobinostat $30 \mathrm{mg}$ and carfilzomib $20 / 45 \mathrm{mg} / \mathrm{m}^{2}$.

The ORR was $67 \%$ and CBR $79 \%$ for all patients. For patients treated at the MTD, ORR was $72 \%$ and CBR $88 \%$. Median PFS was 7.7 months, with a median time to progression of 7.7 months. Median OS was not reached: at 24 months, OS was 67\%. Grade 3/4 hematologic AEs were thrombocytopenia (38\%), neutropenia (21\%), and anemia (9\%). Nonhematologic grade 3 and 4 AEs were fatigue (11\%) and hypertension (9\%). Neuropathy grade 3 or 4 did not occur. In total, 14 serious treatment-related AEs occurred in 12 patients. One patient died due to treatment-related heart failure. Three patients discontinued treatment due to toxicity of treatment: anemia, decreased left ventricular ejection fraction, and supraventricular tachycardia. ${ }^{30}$

Another phase I dose-finding study was performed by Kaufman et al, combining panobinostat and carfilzomib. Panobinostat was administered three times a week in the first 3 weeks of a 4-week cycle at a dose ranging from $15 \mathrm{mg}$ to 20 mg. Carfilzomib was administered twice weekly during the first 3 weeks at doses ranging from $20 / 27 \mathrm{mg} / \mathrm{m}^{2}$ to $20 / 45 \mathrm{mg}$ / $\mathrm{m}^{2}$. The MTD was panobinostat $20 \mathrm{mg}$ in combination with carfilzomib $20 / 36 \mathrm{mg} / \mathrm{m}^{2}$. In this study, three DLTs occurred: grade 4 thrombocytopenia with grade 3 acute kidney injury, persistent grade 4 thrombocytopenia and grade 3 diarrhea. Grade 3 or higher anemia (35\%), thrombocytopenia (35\%), neutropenia $(20 \%)$, fatigue $(15 \%)$, anorexia $(10 \%)$, hyponatremia $(10 \%)$, and nausea $(10 \%)$ occurred. The ORR was $50 \%$, with a median PFS of 14.3 months. ${ }^{31}$

Vesole et al investigated the combination of carfilzomib (15 mg, $20 \mathrm{mg}$, or $20 / 27 \mathrm{mg} / \mathrm{m}^{2}$ on days $1,2,8,9,15$, and 22 ), lenalidomide (15 or $25 \mathrm{mg}$ from day 1 until day 21), vorinostat (300 or $400 \mathrm{mg}$, days 1-7 and 15-21), and dexamethasone $40 \mathrm{mg}$ weekly. A total of 17 patients were treated in this phase I study, in which no DLT was reported and the MTD not reached. Grade 3 or higher AEs were anemia (41\%), thrombocytopenia (53\%), neutropenia (53\%), infection (18\%), hyperglycemia (18\%), electrolyte imbalance (12\%), fatigue (6\%), and constipation (6\%). There were no cardiac AEs. Muscle cramping occurred in 59\% of patients, though all grade 2 or lower. The ORR was 53\%, with $12 \%$ achieving a very good PR and $41 \%$ a PR. Median time to response for these patients was 2 months, with median DOR of 15 months and $29 \%$ of patients achieving stable disease. Median PFS was 12 months where median OS was not reached. ${ }^{32}$

\section{Carfilzomib, pomalidomide and dexamethasone}

Shah et al investigated the MTD of carfilzomib, pomalidomide, and dexamethasone in a phase I study in RRMM patients with a median six prior lines of therapy. In total, 32 patients were enrolled. Initial doses were carfilzomib 20/27 $\mathrm{mg} / \mathrm{m}^{2}$, with pomalidomide once daily from day 1 till day 21 starting at $4 \mathrm{mg}$ and dexamethasone $40 \mathrm{mg} /$ week. All patients were refractory to lenalidomide. A total of 32 of 33 patients received bortezomib, of which 30 were refractory to bortezomib. The MTD was determined at dose level 1, as described earlier. ORR was $50 \%$, CBR $66 \%$, and $16 \%$ of patients reached a very good PR. Median PFS was 7.2 months and median OS 20.6 months. Grade 3 or higher AEs were, among others, acute renal failure (three patients), congestive heart failure (one patient), pneumonia (four patients), and dyspnea (one patient). Two patients experienced pulmonary embolism, even though they used aspirin prophylaxis. There was no grade 3 or higher PNP. ${ }^{33}$

\section{Carfilzomib, ibrutinib and dexamethasone}

Chari et al conducted a phase I trial investigating ibrutinibcarfilzomib-dexamethasone combination therapy in patients with RRMM. The primary objective was to determine the MTD. Secondary objectives were to determine ORR and DOR. Ibrutinib was prescribed at $560 \mathrm{mg}$ or $840 \mathrm{mg}$ once daily from day 8 in the first cycle in a 28-day cycle. Carfilzomib was administered twice weekly in the first 3 weeks, starting at $20 \mathrm{mg} / \mathrm{m}^{2}$ and increased to 27 or $36 \mathrm{mg} / \mathrm{m}^{2}$. Dexamethasone was used orally at $20 \mathrm{mg}$ /day twice weekly. A total of 43 patients participated, and no DLT was observed. AEs of grade 3 or higher occurred in $86 \%$ of patients, including hematologic toxicities (thrombocytopenia 12\%, neutropenia 7\%, anemia 19\%) and nonhematologic AEs (hypertension $23 \%$, pneumonia $19 \%$, fatigue $16 \%$, diarrhea $14 \%$ ). Two patients experienced grade 3 PNP. A total of 13 patients discontinued treatment, of which 4 experienced a cardiac event ( 1 heart failure, 2 atrial fibrillation, 1 atrial flutter). The ORR was $67 \%$ and CBR 76\%. Median DOR was 12.9 months. Even in bortezomib-refractory patients, ORR was $73 \%$, with median DOR 9.1 months. Comparable ORR of $78 \%$ and DOR were measured in patients with high-risk cytogenetics (del17p and/or t[4;14]).

Median PFS was 7.2 months at a median follow-up of 20.5 months. In high-risk patients, PFS was 8.1 months. The authors concluded that the combination of ibrutinib and carfilzomib with dexamethasone is a possible treatment modality, with promising responses rates that are durable. ${ }^{34}$ The initial results of the phase II part of the study were recently published, 
in which 40 patients were treated at the recommended phase II dose, with ORR of $72 \%$ and median PFS of 11.6 months. For high-risk and non-high-risk patients, ORR and PFS were $75 \%$ and 8.1 months vs $71 \%$ and 9.4 months, respectively, suggesting that this treatment combination might overcome the adverse prognosis of high-risk cytogenetic MM. Grade 3 or higher AEs that occurred were anemia (15\%), thrombocytopenia $(13 \%)$, neutropenia ( $8 \%)$, hypertension $(18 \%)$, pneumonia $(13 \%)$, diarrhea $(10 \%)$, and hyperglycemia $(10 \%) .{ }^{35}$

\section{Carfilzomib, isatuximab and dexamethasone}

In a phase IB study, isatuximab was combined with carfilzomib in RRMM. In total, 33 patients were treated in the dose-escalation and dose-expansion cohort, with 29 evaluable patients. ORR was $61 \%$ and CBR $86 \%$. Median PFS was not reached, and there was no DLT or severe toxicity. Based on these promising results, the phase III IKEMA study was initiated, which randomized patients with RRMM between carfilzomib and dexamethasone vs carfilzomib and dexamethasone combined with isatuximab (anti-CD38). Estimated study completion is April 2019. ${ }^{36}$

\section{Carfilzomib, daratumumab and dexamethasone}

Recently, a subgroup analysis of the MMY1001 study (phase IB) was published, in which daratumumab (anti-CD38) was combined with carfilzomib and dexamethasone in lenalidomide-refractory patients with RRMM. A total of 51 lenalidomide-refractory patients were treated and showed an ORR of $81 \%$, with 8 patients achieving maximum recommended dose-negative status. The ORR for all patients was $86 \%$. The most common grade 3 or 4 AEs were thrombocytopenia (37\%), anemia (29\%), neutropenia (28\%), and lymphopenia $(26 \%) .{ }^{37}$ In the CANDOR study, the addition of daratumumab to carfilzomib and dexamethasone is being investigated in a phase III randomized study in RRMM patients with one to three prior lines of treatment.

\section{Selinexor, carfilzomib and dexamethasone}

Currently, a phase I study in which selinexor, carfilzomib, and dexamethasone are to be combined is enrolling patients to determine the MTD and recommended phase II dose. Treatment with carfilzomib-based regimens can be quite intensive for patients, coming to the hospital twice weekly for 3 weeks in a row in a 4-week cycle. This burden has led to a phase I/ II study investigating the possibility of a once-weekly dosing schedule of carfilzomib combined with dexamethasone.

\section{Carfilzomib in once-weekly administration schedules}

In the phase I part of the CHAMPION-1 study, the MTD was established at $70 \mathrm{mg} / \mathrm{m}^{2}$ weekly with 30 -minute infusions on days 1,8 , and 15 of a 28 -day cycle. In the phase II part of the study, 89 patients were treated with carfilzomib $70 \mathrm{mg} / \mathrm{m}^{2}$ in combination with dexamethasone $40 \mathrm{mg}$ once weekly. In total, 104 patients received carfilzomib at $70 \mathrm{mg} /$ $\mathrm{m}^{2}$ once weekly. The median duration of treatment was 7.7 months, ORR was $77 \%, 13 \%$ of patients achieved a complete response, and CBR was $84 \%$. The ORR for bortezomibexposed and -refractory and lenalidomide-refractory patients was $73 \%, 63 \%$, and $71 \%$ respectively. Median PFS was 12.6 months.

In total, $62 \%$ of patients experienced grade 3 or higher AEs: thrombocytopenia (6\%), anemia (6\%), neutropenia (4\%), fatigue (11\%), hypertension (7\%), pneumonia $(6 \%)$, and acute kidney injury (6\%). Grade 3 cardiac failure was seen in $2 \%$ of patients, and grade 3 PNP in only $1 \%$. Five patients died during the study, one due to disease progression and four due to AEs. Two of these - acute respiratory distress syndrome and cardiopulmonary arrest - were possibly carfilzomib-related. This schedule might be beneficial, considering the lower burden for patients and caregivers, with comparable toxicity to the twice-weekly administration schedule. ${ }^{38}$

This study led to a phase III study (ARROW), published in 2018, in which RRMM patients with two or three previous lines of treatments, including a PI (carfilzomib or oprozomib excluded) and an IMiD, were treated with once- $(20 / 70 \mathrm{mg} /$ $\mathrm{m}^{2}$ ) or twice-weekly carfilzomib $\left(20 / 27 \mathrm{mg} / \mathrm{m}^{2}\right)$, with weekly dexamethasone dosed at $40 \mathrm{mg}$. A total of 478 patients were included in the study. Median PFS was 11.2 months for the once-weekly group and 7.6 months for the twice-weekly group ( $P=0.0029$ ), with time to progression of 12.4 months and 8.5 months, respectively. ORR was $62.9 \%$ in the onceweekly group vs $40.8 \%$ in the twice-weekly group, with median DOR of 15.0 and 13.8 months and median time to response of 1.1 and 1.9 months. Median OS was not reached. At 12 months, OS was $76.6 \%$ for the once-weekly group vs $71.9 \%$ for the twice-weekly group.

AEs of grade 3 or higher occurred in $68 \%$ of patients in the once-weekly group vs $62 \%$ in the twice-weekly group. Hematologic grade 3 or higher AEs were anemia (18\% vs $18 \%$ ), thrombocytopenia ( $7 \%$ vs $7 \%$ ), and neutropenia ( $6 \%$ vs 7\%). Nonhematologic AEs of grade 3 or higher included pneumonia ( $10 \%$ vs $7 \%$ ), hypertension ( $6 \%$ vs $5 \%$ ), and sepsis $(3 \%$ vs $1 \%$ ). No grade 3 or higher PNP occurred in 
the once-weekly group and in only one person in the twiceweekly group, $3 \%$ of patients in the once-weekly group had grade 3 or higher cardiac failure vs $4 \%$ in the twice-weekly group, and $13 \%$ of patients in the once-weekly group and $12 \%$ of patients in the twice-weekly group had to discontinue carfilzomib due to a treatment-emergent AE. ${ }^{13}$ Based on the results of the ARROW study, recently the once-weekly administration of carfilzomib has been approved by the FDA for patients with RRMM.

\section{Toxicity of carfilzomib Single agent}

Siegel et al reviewed the safety profile of carfilzomib as a single agent where data from four phase II studies (PX171-003-A0 and A1, PX-171-004, and PX-171-005) were combined. In total, 526 patients were included in the analysis: $14.6 \%$ of patients required a dose reduction and $22.6 \%$ required a dose delay due to an $\mathrm{AE}$, while $14.8 \%$ of patients discontinued therapy due to an AE. Reasons to discontinue therapy were congestive heart failure $(1.5 \%)$, dyspnea $(1.3 \%)$, acute renal failure/increased blood creatinine (both 1.1\%), and cardiac arrest (1.0\%). In the following sections, the most important AEs are described. Cardiac AEs are discussed separately. The AEs of any grade most reported overall were fatigue (55\%), anemia (46.8\%), and nausea (44.9\%).

\section{Hematologic}

The most reported grade 3 or higher AEs were thrombocytopenia (23.4\%), anemia (22.4\%), lymphopenia (18.1\%), and pneumonia $(10.5 \%)$.

\section{Renal}

In sum, $23.8 \%$ of patients had creatinine clearance $<50 \mathrm{~mL} /$ $\mathrm{min}, 39.4 \%$ had clearance of $50-80 \mathrm{~mL} / \mathrm{min}$ at baseline, and $13.2 \%$ had at least one episode of decrease in renal function of any grade. In half of these patients, the decline in renal function was transient, and the median duration of worsening of renal clearance was 1.4 weeks. In the other 37 patients, renal function decline was nontransient, and 8 of these 37 discontinued treatment due these renal side effects.

\section{Peripheral neuropathy}

At baseline, $71.9 \%$ of patients experienced PNP grade 1 or grade 2 . In this pooled analysis, $1.3 \%$ of carfilzomib-treated patients experienced deterioration to grade 3 PNP. All these patients had grade 1 or 2 PNP at baseline. No grade 4 PNP was reported. Only one patient had to discontinue treatment due to neuropathic pain.

\section{Pulmonary}

In sum, $42.2 \%$ of patients reported dyspnea and $26.0 \%$ cough. Most were grade 1 or 2 . Grade 3 AEs were reported by $4.8 \%$ of patients. There were no grade 4 pulmonary AEs. One patient died due to congestive heart failure, and $1.3 \%$ of patients discontinued treatment due to a pulmonary AE. Other AEs were pleural effusion (4\%), pulmonary hypertension $(2 \%)$, pulmonary embolism $(1 \%)$, hemoptysis $(0.6 \%)$, and pneumonitis $(0.4 \%)$, while $18.8 \%$ of patients had at least one respiratory infection. Two patients died due to respiratory infection.

\section{Tumor-lysis syndrome}

In total, five patients experienced TLS. After prophylactic guidelines were implemented, only one patient experienced TLS (of five in total). ${ }^{39}$ In the pooled analysis of 526 patients receiving carfilzomib monotherapy $7.2 \%$ experienced cardiac failure during treatment and $22.1 \%$ reported any cardiac AE, including $14.3 \%$ hypertension. The cardiovascular event rate described was higher than previously described with treatment with bortezomib. The exact underlying mechanism of cardiac AEs is still unknown. In the following section, some pathophysiological background is discussed. ${ }^{39}$

\section{Cardiovascular AEs and possible pathophysiological mechanisms}

Cardiac events that have been observed in several studies include hypertension, congestive heart failure, and coronary artery disease. The exact underlying pathophysiological mechanism is unknown. Whether it is due to an endothelial effect, accumulation of misfolded proteins in cardiomyocytes, short infusion time, higher dosage of carfilzomib, or a combination of these possible mechanisms needs to be cleared up.

To investigate whether carfilzomib has a damaging effect on cardiac endothelial cells, Chen-Scarabelli et al administered carfilzomib to isolated rabbit hearts and aortae. They found that carfilzomib increased coronary perfusion pressure, resting vasoconstriction tone, and the spasmogenic effect of other different agents. Carfilzomib also reduced the vasodilating effect of acetylcholine, suggesting carfilzomib has an impaired vasodilating effect via an endothelium-dependent mechanism..$^{40}$

PIs induce cell death of MM cells due to accumulation of misfolded of unfolded proteins, leading to cytotoxic stress and apoptosis. Fu et al exposed rat neonatal cardiomyocytes to PIs (MG132, epoxomicin), leading to endoplasmic reticulum-initiated death of cardiomyocytes. This might 
also be one of the explanations for the use of PIs sometimes leading to cardiac failure. ${ }^{41}$ Another factor of importance might be the infusion time of and dosage of carfilzomib. In a single-center retrospective analysis of 130 patients treated with carfilzomib, 26 patients developed significant cardiac AEs, with 20 of 26 patients receiving the carfilzomib in 2- to 10-minute infusions. ${ }^{42}$

Land et al performed a retrospective analysis of patients receiving bortezomib and/or carfilzomib from 2010 until 2014, investigating the incidence of cardiac AEs. A total of 157 patients were included: 47 treated with bortezomib and 110 with carfilzomib. In sum, 17\% reported cardiotoxicity: $9 \%$ in the bortezomib and $20 \%$ in the carfilzomib group. Baseline cardiac characteristics of value to predict cardiac AEs were not found. However, there was a significant increase in cardiac AEs in the group treated at $\geq 36 \mathrm{mg} / \mathrm{m}^{2}$ compared to $<20 \mathrm{mg} / \mathrm{m}^{2}$ and $27 \mathrm{mg} / \mathrm{m}^{2}$. However, patients treated with a dose of $36 \mathrm{mg} / \mathrm{m}^{2}$ were more heavily pretreated MM patients. ${ }^{43}$

Is monitoring of cardiovascular parameters before and during treatment of any additive value? Dimopoulos et al investigated the possible cardiotoxic effects of carfilzomib in 60 patients by performing serial echocardiography: $12 \%$ of patients experienced a reduction in left ventricular ejection fraction, which increased in time from $5 \%$ at 3 months till $12 \%$ at 15 months, and $23.5 \%$ of patients with a previous cardiovascular disease had a decline in left ventricular ejection fraction compared to only $7 \%$ in patients without previous cardiovascular disease. There was no association between the dose of carfilzomib or the duration of infusion. Also, a transient decrease in kidney function was noticed, although $55 \%$ of patients with baseline estimated glomerular filtration rate $<60 \mathrm{~mL} / \mathrm{min} / 1.73 \mathrm{~m}^{2}$ showed an improved renal function. ${ }^{44}$

In a prospective study investigating 62 patients treated with carfilzomib (first-line treatment as well as RRMM treatment), blood pressure, N-terminal pro-brain natriuretic peptide, echocardiography, and kidney function were monitored. Cardiovascular events were infrequent, but more frequently seen in heavily pretreated patients and those with cardiovascular dysfunction at baseline. ${ }^{45}$ Even though the pathophysiological mechanisms of cardiovascular events during carfilzomib treatment are not completely unraveled, caution is clearly needed in cardiovascularly compromised patients when treatment with carfilzomib is started.

\section{Toxicity in combination therapy}

The ENDEAVOR study, combining carfilzomib and dexamethasone, and the ASPIRE study, adding lenalidomide to this combination, are the only completed phase III studies performed with carfilzomib. Table 2 shows an overview of the most common hematologic and nonhematologic AEs in these two studies. It is notable that grade III or higher hematologic AEs in the ENDEAVOR and ASPIRE studies were lower compared to the pooled analysis of the AEs of carfilzomib as a single agent.

\section{Place in therapy}

Patients with RRMM who have received at least one prior therapy are potential candidates for treatment with carfilzomib. Currently, carfilzomib (dosed at $20 / 56 \mathrm{mg} / \mathrm{m}^{2}$ ) combined with dexamethasone and once-weekly dosing of carfilzomib $\left(20 / 70 \mathrm{mg} / \mathrm{m}^{2}\right)$ combined with dexamethasone and carfilzomib in combination with lenalidomide and dexamethasone are FDA-approved (carfilzomib dosed at $20 / 27 \mathrm{mg} / \mathrm{m}^{2}$ ) for RRMM.

Several (combination) treatments are available for RRMM nowadays, as (next-generation) IMiDs, (next-generation) PIs, alkylating agents, monoclonal antibodies, for example. Comprehensive assessment of prior therapies, prior toxicity, and weighting the different therapeutic options available are important determinants in choosing a subsequent therapy. The goal is to provide a treatment option that differs from frontline and/or other prior salvage regimens, with potent myeloma activity and a favorable toxicity profile.

\section{Recommendations for daily practice}

In the first administrations of carfilzomib, higher amounts of intravenous fluids (at least $250 \mathrm{~mL}$ ), in combination with rasburicase in case of high tumor load, high levels of urate or preemptive renal impairment is advised to prevent TLS. Monitor blood chemistries closely, particularly in cycle 1 , to anticipate for TLS. In our practice, this currently means obtaining laboratory reports on days $1,2,8$, and 15 in cycle 1 and before the start of each cycle thereafter. Prophylaxis with allopurinol is not advised, due to possible interactions. Intravenous fluids $(250 \mathrm{~mL})$ are recommended before each dose of carfilzomib in the first cycle if the patient is able to tolerate such a fluid challenge. In cycle 2 and beyond, $100 \mathrm{~mL}$ of intravenous fluid or even oral hydration is likely adequate.

Cardiac AEs are thought to be a class effect of PIs, because they have been reported with bortezomib, carfilzomib and ixazomib; however, they appear to be more frequent with carfilzomib. At present, there are no strategies to prevent cardiovascular events that have been validated in prospective studies. However, as advised by the European Myeloma Network, cardiovascular risks should be assessed and wherever 
Table 2 Toxicity data/adverse events of ENDEAVOR and ASPIRE studies

\begin{tabular}{|c|c|c|c|c|c|c|c|c|}
\hline & \multicolumn{4}{|l|}{ ASPIRE } & \multicolumn{4}{|c|}{ ENDEAVOR } \\
\hline & \multicolumn{2}{|c|}{ Len-Dex-Car $(n=392)$} & \multicolumn{2}{|c|}{ Len-Dex $(n=389)$} & \multicolumn{2}{|c|}{ Car-Dex $(n=463)$} & \multicolumn{2}{|c|}{ Bor-Dex $(n=456)$} \\
\hline & All grades & Grade $\geq 3$ & All grades & Grade $\geq 3$ & All grades & Grade $\geq 3$ & All grades & Grade $\geq 3$ \\
\hline \multicolumn{9}{|l|}{ Hematological } \\
\hline Anemia & 42.6 & 17.9 & 39.8 & 17.2 & 42 & 16 & 28 & 10 \\
\hline Thrombocytopenia & 29.1 & 16.6 & 22.6 & 12.3 & 22 & 9 & 18 & 9 \\
\hline Neutropenia & 37.8 & 29.6 & 33.7 & 26.5 & & & & \\
\hline Lymphopenia & & & & & 7 & 5 & 5 & 3 \\
\hline \multicolumn{9}{|l|}{ Nonhematological } \\
\hline Diarrhea & 42.3 & 3.8 & 33.7 & 4.1 & 36 & 4 & 41 & 9 \\
\hline Fatigue & 32.9 & 7.7 & 30.6 & 6.4 & 32 & 7 & 31 & 8 \\
\hline Cough & 28.8 & 0.3 & 17.2 & 0 & 28 & 0 & 16 & $<1$ \\
\hline Pyrexia & 28.6 & 1.8 & 20.8 & 0.5 & 32 & 3 & 15 & 1 \\
\hline Upper respiratory tract infection & 28.6 & 1.8 & 19.3 & I & 26 & 2 & 18 & $\mathrm{I}$ \\
\hline Hypokalemia & 27.6 & 9.4 & 13.4 & 4.9 & 13 & 2 & 11 & 4 \\
\hline Muscle spasms & 26.5 & I & 21.1 & 0.8 & 20 & $<1$ & 6 & I \\
\hline Peripheral edema & 21.7 & 1.3 & 19.3 & 0.5 & 25 & $\mathrm{I}$ & 19 & 1 \\
\hline Nasopharyngitis & 21.4 & 0.3 & 16.2 & 0 & 17 & $<1$ & 13 & $<1$ \\
\hline Constipation & 20.2 & 0.3 & 17.2 & 0.5 & 16 & $<1$ & 28 & 2 \\
\hline Back pain & 17.1 & 1.3 & 20.1 & 2.1 & 23 & 2 & 18 & 3 \\
\hline Dyspnea & 19.4 & 2.8 & 14.9 & 1.8 & 32 & 6 & 14 & 2 \\
\hline Peripheral neuropathy & 17.1 & 2.6 & 17 & 3.1 & 11 & $\mathrm{I}$ & 29 & 6 \\
\hline Hypertension & 14.3 & 4.3 & 6.9 & 1.8 & 32 & 14 & 10 & 3 \\
\hline Acute renal failure & 8.4 & 3.3 & 7.2 & 3.1 & & & & \\
\hline Elevated creatinine & 6.6 & $\mathrm{I}$ & 4.6 & 0.3 & 11 & 1 & 6 & $<1$ \\
\hline Cardiac failure & 6.4 & 3.8 & 4.1 & 1.8 & 5 & 3 & 1 & I \\
\hline Deep-vein thrombosis & 6.6 & 1.8 & 3.9 & $\mathrm{I}$ & & & & \\
\hline Ischemic heart disease & 5.9 & 3.3 & 4.6 & 2.1 & & & & \\
\hline Pulmonary embolism & 3.6 & 3.1 & 2.3 & 2.3 & & & & \\
\hline
\end{tabular}

Note: All numbers are percentages.

Abbreviations: Bor, bortezomib; Car, carfilzomib; Dex, dexamethasone; Len, lenalidomide.

possible corrected before starting therapy with carfilzomib. ${ }^{46}$ Therefore, it is advised to measure and correct modifiable risk factors, such as hypertension, high cholesterol levels, hyperglycemia, tobacco use, and incorrect diet. Patients with cardiac risks may benefit from a cardiology review prior to receiving treatment, and should be closely monitored for fluid overload. Before and during treatment, regular clinical surveillance with blood-pressure control and tight treatment of hypertension with antihypertensives is recommended, pursuing normotension during treatment with carfilzomib.

Unfortunately, a good screening method to identify patients at risk for cardiac events is not yet available. Serial monitoring of cardiac function via echocardiography or cardiac biomarkers, such as $\mathrm{N}$-terminal pro-brain natriuretic peptide are considered of limited value in mitigating the risk of carfilzomib-associated cardiac failure. ${ }^{45}$ In the event of grade 3 or 4 cardiac events, carfilzomib should be withheld until recovery. ${ }^{42}$ Carfilzomib may be resumed at the physician's discretion based on a benefit-risk assessment, although preferably at a reduced dose. After the first cycle, caution is advised with regard to administering large amounts of intravenous fluid, especially in cardiac-compromised, hypertensive, and elderly patients. All patients should be routinely controlled for fluid overload. Furthermore, physicians should also be aware of the possible development of pulmonary hypertension.

Dose modifications in patients with baseline renal impairment are not necessary. However, during treatment renal function should be routinely evaluated at the start of each cycle and whenever indicated, as sudden renal insufficiency has been described. Then, further carfilzomib should be discontinued. Carfilzomib is not metabolized via the liver. Therefore, mild liver impairment is no contraindication for treatment; however, patients with severe liver impairment have been excluded from clinical trials. As such, we advise careful monitoring in patients with severe liver impairment.

Immunomodulatory drugs, especially in combination with corticosteroids and chemotherapy, are associated with 
high frequency of thromboembolic complications. Therefore, for the combination of carfilzomib with lenalidomide and dexamethasone, prophylaxis with cardioaspirin, low-molecular-weight heparin, or warfarin is mandatory. The choice of the most appropriate drug depends on the thrombotic risk: if the probability of venous thromboembolism/pulmonary embolism is high, low-molecular-weight heparin or warfarin should be preferred. Routine evaluations of normal blood levels, especially platelet counts, are the standard of care. Also, as with bortezomib treatment, herpes zoster prophylaxis is recommended.

\section{Conclusion}

Carfilzomib is a potent selective PI that irreversibly binds to the proteasome. Carfilzomib is generally well tolerated, and seems to be a safe treatment option in patients with renal impairment, patients with liver impairment, and patients with PNP. However, a slightly higher incidence of cardiovascular toxicity is seen, for which further tools for identifying patients at risk are necessary. Altogether, carfilzomib is a welcome addition to the therapeutic arsenal for RRMM. Importantly, a more patient-friendly regimen, dosing once a week instead of twice a week, seems feasible, and has recently been FDAapproved for carfilzomib monotherapy. Ongoing phase II and III trials will help further to define the role of carfilzomib in frontline treatment and the sequence of (combination) treatment schedules in relapsed MM, as well as helping to establish best dosing schemes and supportive-care management during treatment with carfilzomib, further benefiting MM patients.

\section{Disclosure}

NWCJD and SZ have received compensation as part of the advisory board of Amgen. The other authors report no conflicts of interest in this work.

\section{References}

1. Kumar SK, Dispenzieri A, Lacy MQ, et al. Continued improvement in survival in multiple myeloma: changes in early mortality and outcomes in older patients. Leukemia. 2014;28(5):1122-1128.

2. Kumar SK, Dimopoulos MA, Kastritis E, et al. Natural history of relapsed myeloma, refractory to immunomodulatory drugs and proteasome inhibitors: a multicenter IMWG study. Leukemia. 2017;31(11):2443-2448.

3. Richardson PG, Sonneveld P, Schuster MW, et al. Bortezomib or highdose dexamethasone for relapsed multiple myeloma. $N$ Engl J Med. 2005;352(24):2487-2498.

4. Demo SD, Kirk CJ, Aujay MA, et al. Antitumor activity of PR-171, a novel irreversible inhibitor of the proteasome. Cancer Res. 2007;67(13):6383-6391.

5. Leleu X, Martin TG, Einsele H, et al. Role of proteasome inhibitors in relapsed and/or refractory multiple myeloma. Clin Lymphoma Myeloma Leuk. 2018;19(1):9-22.
6. Guerrero-Garcia TA, Gandolfi S, Laubach JP, et al. The power of proteasome inhibition in multiple myeloma. Expert Rev Proteomics. 2018;15(12):1033-1052.

7. Adams J. The proteasome: a suitable antineoplastic target. Nat Rev Cancer. 2004;4(5):349-360.

8. Niewerth D, Jansen G, AssarafYG, Zweegman S, Kaspers GJL, Cloos J. Molecular basis of resistance to proteasome inhibitors in hematological malignancies. Drug Resist Updat. 2015;18:18-35.

9. Kuhn DJ, Chen Q, Voorhees PM, et al. Potent activity of carfilzomib, a novel, irreversible inhibitor of the ubiquitin-proteasome pathway, against preclinical models of multiple myeloma. Blood. 2007;110(9):3281-3290.

10. Parlati F, Lee SJ, Aujay M, et al. Carfilzomib can induce tumor cell death through selective inhibition of the chymotrypsin-like activity of the proteasome. Blood. 2009;114(16):3439-3447.

11. Arastu-Kapur S, Anderl JL, Kraus M, et al. Nonproteasomal targets of the proteasome inhibitors bortezomib and carfilzomib: a link to clinical adverse events. Clin Cancer Res. 2011;17(9):2734-2743.

12. Yang J, Wang Z, Fang Y, et al. Pharmacokinetics, pharmacodynamics, metabolism, distribution, and excretion of carfilzomib in rats. Drug Metab Dispos. 2011;39(10):1873-1882.

13. Moreau P, Mateos MV, Berenson JR, et al. Once weekly versus twice Weekly carfilzomib dosing in patients with relapsed and refractory multiple myeloma (A.R.R.O.W.): interim analysis results of a randomised, phase 3 study. The Lancet Oncol. 2018;19(7):953-964.

14. O'Connor OA, Stewart AK, Vallone M, et al. A phase 1 dose escalation study of the safety and pharmacokinetics of the novel proteasome inhibitor carfilzomib (PR-171) in patients with hematologic malignancies. Clin Cancer Res. 2009;15(22):7085-7091.

15. Alsina M, Trudel S, Furman RR, et al. A phase I single-agent study of twice-weekly consecutive-day dosing of the proteasome inhibitor carfilzomib in patients with relapsed or refractory multiple myeloma or lymphoma. Clin Cancer Res. 2012;18(17):4830-4840.

16. Siegel DS, Martin T, Wang M, et al. A phase 2 study of single-agent carfilzomib (PX-171-003-A1) in patients with relapsed and refractory multiple myeloma. Blood. 2012;120(14):2817-2825.

17. Vij R, Wang M, Kaufman JL, et al. An open-label, single-arm, phase 2 (PX-171-004) study of single-agent carfilzomib in bortezomib-naive patients with relapsed and/or refractory multiple myeloma. Blood. 2012;119(24):5661-5670.

18. Vij R, Siegel DS, Jagannath S, et al. An open-label, single-arm, phase 2 study of single-agent carfilzomib in patients with relapsed and/or refractory multiple myeloma who have been previously treated with bortezomib. Br J Haematol. 2012;158(6):739-748.

19. Badros AZ, Vij R, Martin T, et al. Carfilzomib in multiple myeloma patients with renal impairment: pharmacokinetics and safety. Leukemia. 2013;27(8):1707-1714.

20. Hájek R, Masszi T, Petrucci MT, et al. A randomized phase III study of carfilzomib vs low-dose corticosteroids with optional cyclophosphamide in relapsed and refractory multiple myeloma (Focus). Leukemia. 2017;31(1):107-114.

21. Papadopoulos KP, Siegel DS, Vesole DH, et al. Phase I study of 30-minute infusion of carfilzomib as single agent or in combination with low-dose dexamethasone in patients with relapsed and/or refractory multiple myeloma. J Clin Oncol. 2015;33(7):732-739.

22. Lendvai N, Hilden P, Devlin S, et al. A phase 2 single-center study of carfilzomib $56 \mathrm{mg} / \mathrm{m} 2$ with or without low-dose dexamethasone in relapsed multiple myeloma. Blood. 2014;124(6):899-906.

23. Dimopoulos MA, Goldschmidt H, Niesvizky R, et al. Carfilzomib or bortezomib in relapsed or refractory multiple myeloma (endeavor): an interim overall survival analysis of an open-label, randomised, phase 3 trial. Lancet Oncol. 2017;18(10):1327-1337.

24. Dimopoulos MA, Moreau P, Palumbo A, et al; ENDEAVOR Investigators. Carfilzomib and dexamethasone versus bortezomib and dexamethasone for patients with relapsed or refractory multiple myeloma (endeavor): a randomised, phase 3, open-label, multicentre study. Lancet Oncol. 2016;17(1):27-38. 
25. Niesvizky R, Martin TG, Bensinger W III, et al. Phase Ib dose-escalation study (PX-171-006) of carfilzomib, lenalidomide, and low-dose dexamethasone in relapsed or progressive multiple myeloma. Clin Cancer Res. 2013;19(8):2248-2256.

26. Wang M, Martin T, Bensinger W, et al. Phase 2 dose-expansion study (PX-171-006) of carfilzomib, lenalidomide, and low-dose dexamethasone in relapsed or progressive multiple myeloma. Blood. 2013;122(18):3122-3128.

27. Stewart AK, Rajkumar SV, Dimopoulos MA, et al; ASPIRE Investigators. Carfilzomib, lenalidomide, and dexamethasone for relapsed multiple myeloma. N Engl J Med. 2015;372(2):142-152.

28. Siegel DS, Dimopoulos MA, Ludwig H, et al. Improvement in overall survival with carfilzomib, lenalidomide, and dexamethasone in patients with relapsed or refractory multiple myeloma. J Clin Oncol. 2018;36(8):728-734.

29. Hideshima T, Richardson PG, Anderson KC. Mechanism of action of proteasome inhibitors and deacetylase inhibitors and the biological basis of synergy in multiple myeloma. Mol Cancer Ther. 2011;10(11):2034-2042.

30. Berdeja JG, Hart LL, Mace JR, et al. Phase I/II study of the combination of panobinostat and carfilzomib in patients with relapsed/refractory multiple myeloma. Haematologica. 2015;100(5):670-676.

31. Kaufman JL, Zimmerman T, Rosenbaum CA, et al. Phase I study of the combination of carfilzomib and panobinostat for patients with relapsed and refractory myeloma: a multiple myeloma research Consortium (MMRC) clinical trial. Blood. 2014;124(21):32.

32. Vesole DH, Bilotti E, Richter JR, et al. Phase I study of carfilzomib, lenalidomide, vorinostat, and dexamethasone in patients with relapsed and/or refractory multiple myeloma. Br J Haematol. 2015;171(1): 52-59.

33. Shah JJ, Stadtmauer EA, Abonour R, et al. Carfilzomib, pomalidomide, and dexamethasone for relapsed or refractory myeloma. Blood. 2015;126(20):2284-2290.

34. Chari A, Larson S, Holkova B, et al. Phase 1 trial of ibrutinib and carfilzomib combination therapy for relapsed or relapsed and refractory multiple myeloma. Leuk Lymphoma. 2018;59(11):2588-2594.

35. Chari A, Girnius S, Chhabra S, et al. Initial phase 2 results of ibrutinib combined with carfilzomib/dexamethasone in patients with relapsed/ refractory multiple myeloma. Blood. 2017;130(Suppl 1):3111.
36. Chari A, Richter JR, Shah N, et al. Phase I-B study of isatuximab + carfilzomib in relapsed and refractory multiple myeloma (RRMM). $J$ Clin Oncol. 2018;36(15_suppl):8014.

37. Chari A, Martinez-Lopez J, Mateos MV, et al. Daratumumab (DARA) in combination with carfilzomib and dexamethasone (D-Kd) in lenalidomide (Len)-refractory patients (Pts) with relapsed multiple myeloma (MM): Subgroup analysis of MMY1001. J Clin Oncol. 2018;36(15_suppl): 8002 .

38. Berenson JR, Cartmell A, Bessudo A, et al. CHAMPION-1: a phase $1 / 2$ study of once-weekly carfilzomib and dexamethasone for relapsed or refractory multiple myeloma. Blood. 2016;127(26):3360-3368.

39. Siegel D, Martin T, Nooka A, et al. Integrated safety profile of singleagent carfilzomib: experience from 526 patients enrolled in 4 phase II clinical studies. Haematologica. 2013;98(11):1753-1761.

40. Chen-Scarabelli C, Corsetti G, Pasini E, et al. Spasmogenic effects of the proteasome inhibitor carfilzomib on coronary resistance, vascular tone and reactivity. EBioMedicine. 2017;21:206-212.

41. Fu HY, Minamino T, Tsukamoto O, et al. Overexpression of endoplasmic reticulum-resident chaperone attenuates cardiomyocyte death induced by proteasome inhibition. Cardiovasc Res. 2008;79(4):600-610.

42. Atrash S, Tullos A, Panozzo S, et al. Cardiac complications in relapsed and refractory multiple myeloma patients treated with carfilzomib. Blood Cancer J. 2015;5(1):e272.

43. Land J, Afifi S, Adel NG, et al. Incidence and management of proteasome inhibitor-related cardiotoxicity in multiple myeloma patients at Memorial Sloan Kettering cancer center. Blood. 2015;126(23):4265.

44. Dimopoulos MA, Roussou M, Gavriatopoulou M, et al. Cardiac and renal complications of carfilzomib in patients with multiple myeloma. Blood Adv. 2017;1(7):449-454.

45. Rosenthal A, Luthi J, Belohlavek M, et al. Carfilzomib and the cardiorenal system in myeloma: an endothelial effect? Blood Cancer J. 2016;6(1):e384.

46. Larocca A, Dold SM, Zweegman S, et al. Patient-centered practice in elderly myeloma patients: an overview and consensus from the European myeloma network (EMN). Leukemia. 2018;32(8):1697-1712.

47. Martin TG, Mannis G III, Chari A, Munster P, Campana F, Hui A. Phase Ib study of Isatuximab and carfilzomib in relapse and refractory multiple myeloma. Blood. 2016;128(22):2111.
Cancer Management and Research

\section{Publish your work in this journal}

Cancer Management and Research is an international, peer-reviewed open access journal focusing on cancer research and the optimal use of preventative and integrated treatment interventions to achieve improved outcomes, enhanced survival and quality of life for the cancer patient. The manuscript management system is completely online and includes

\section{Dovepress}

a very quick and fair peer-review system, which is all easy to use. Visit http://www.dovepress.com/testimonials.php to read real quotes from published authors. 